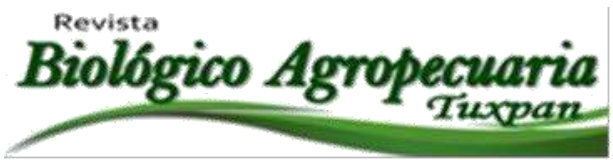

\title{
Calidad de la leche de vaca en una zona de riego del estado de Puebla
}

Quality of cow's milk in an irrigation area of the state of Puebla

\author{
Castro-González Numa Pompilio ${ }^{1 凶}$; Moreno-Rojas Rafael ${ }^{2}$; Calderón-Sánchez Francisco ${ }^{3}$; Escobar Hernández \\ Ramiro $^{1}$ y Barrios Díaz Benjamín ${ }^{1}$
}

${ }^{1}$ Facultad de Ingeniería Agrohidráulica, Benemérita Universidad Autónoma de Puebla, San Juan Acateno, Teziutlán, Puebla, C.P. 73695. ${ }^{2}$ Departamento de Bromatología y Biotecnología de Alimentos, Universidad de Córdoba, España. ${ }^{3}$ Colegio de Postgraduados Campus - Puebla, México.

${ }^{\bowtie}$ Autor para correspondencia: numa.castro@ correo.buap.mx.

Recibido: 08/01/2016 Aceptado: 17/06/2016

\section{RESUMEN}

El objetivo fue determinar la calidad de la leche cruda en base a su composición y a la posible contaminación con metales pesados por la ingestión de alfalfa (Medicago sativa) cultivada en suelos que son irrigados con aguas del canal de riego de Valsequillo en el estado de Puebla. Se muestrearon suelo agrícola, alfalfa y 136 vacas de las cuales se tomaron dos muestras de leche directamente de la ubre, siendo una muestra para calidad en cuanto a su composición y la otra para contenido de metales pesados $(\mathrm{Cd}, \mathrm{Pb}, \mathrm{Cr}$ y $\mathrm{Zn})$. Para el análisis estadístico se utilizó un GLM mediante el paquete estadístico SAS. En este trabajo de encontró diferencia $\mathrm{p}<0.0001$ entre las diferente matrices analizadas, existiendo una concentración mayor en suelo manifestando el siguiente orden decreciente; $\mathrm{Pb}>\mathrm{Cr}>\mathrm{Zn}>\mathrm{Cd},\left(38 ; 31.38 ; 22.78: 2 \mathrm{mg} \mathrm{kg}^{-1}\right)$, seguida de la planta, que tuvo niveles altos. En relación a la leche la concentración promedio obtenida de $\mathrm{Pb}$ fue $0.13 \mathrm{mg} \mathrm{kg}^{-1}$. Además de encontrar niveles considerables de $\mathrm{Cd}, \mathrm{Cr}$ y $\mathrm{Zn}$. En relación la composición de la leche no existió p $>0.05$ entre el tipo de productores, siendo de manera general una leche con bajo porcentaje en grasa. Por tanto se concluye que la leche producida en la región de estudio presenta baja calidad en cuanto a su contenido y considerable nivel de contaminación con metales pesados lo que representa un riesgo para la salud de sus consumidores.

Palabras claves: Metales pesados, leche, cadena alimenticia, suelos contaminados

\begin{abstract}
The objective was to determine the quality of raw milk based on their composition and possible contamination with heavy metals by eating alfalfa (Medicago sativa) grown in soils that are irrigated with water from irrigation canal Valsequillo in the state of Puebla. agricultural land, alfalfa and 136
\end{abstract}


cows which were sampled Two milk samples were taken directly from the udder, being a sample for quality in terms of its composition and the other for content of heavy metals $(\mathrm{Cd}, \mathrm{Pb}, \mathrm{Cr}$ and $\mathrm{Zn})$. For statistical analysis, GLM was used by the SAS statistical package. In this work he found difference $p$ $<0.0001$ between the different matrices analyzed, having a higher concentration in soil stating the following descending order; $\mathrm{Pb}>\mathrm{Cr}>\mathrm{Zn}>\mathrm{Cd}$, $\left(38 ; 31.38 ; 22.78: 2 \mathrm{mg} \mathrm{kg}^{-1}\right)$, followed by the plant, which had high levels. In relation to milk obtained from the average $\mathrm{Pb}$ concentration was $0.13 \mathrm{mg} \mathrm{kg}^{-}$ 1 . Besides finding significant levels of $\mathrm{Cd}, \mathrm{Cr}$ and $\mathrm{Zn}$. Regarding the composition of milk did not exist p> 0.05 between the types of producers, generally being a milk with low fat percentage. Therefore, it has concluded that the milk produced in the study region has low quality in terms of content and significant levels of heavy metal contamination, which poses a risk to the health of their consumers.

Keywords: Heavy metals, milk, food chain, Soil polluted

\section{INTRODUCCIÓN}

En México se producen alrededor de 1.5 millones de litros diarios de leche, siendo ésta en su mayoría bronca, la cual proviene de unidades de producción ubicadas en áreas rurales y periferia de las grandes ciudades, llegando a una cantidad de aproximadamente 100,250 unidades de producción (INEGI/COLPOS,1999; Álvarez-Fuentes et al., 2012). La leche de vaca es uno de los alimentos de mayor valor nutritivo (Soares et al., 2009); recomendado por la FAO y la UNESCO como imprescindible en la dieta, especialmente para niños, por ello es importante cuidar su calidad, la cual está determinada por sus propiedades nutritivas (proteínas, grasa, carbohidratos, minerales y vitaminas), pero también por su proceso de producción. Los factores que afectan la calidad de la leche pueden ser intrínsecos (raza, número y periodo de

lactancia) y extrínsecos (condiciones ambientales, alimentación y manejo sanitario) (Rego et al., 2009; Bobić et al., 2013; Kadlecová et al., 2014; Ducháček et al., 2014). Por tal razón, cuando es producida en condiciones de contaminación puede ser un reservorio de diversos compuestos y dentro de ellos están los metales pesados que son altamente tóxicos, caracterizándose por no biodegradase y causar de graves problemas a la salud pública, debido a que son citotóxicos, cancerígenos y mutagénicos (Oliver, 1997; Sundeberg, et al., 1999; Dorea y Donangelo, 2006; Al-Othman et al., 2012; ATSDR, 2013).

Los metales pesados que aparecen en leche son el resultado de alimentar a las vacas con forrajes o granos provenientes de suelos irrigados con aguas residuales.

Existen trabajos que reportan altos niveles de $\mathrm{Cd}, \mathrm{Hg}, \mathrm{Cr}, \mathrm{Ni}, \mathrm{Pb}$ y As en leche de vacas y cabras alimentadas con productos irrigados con aguas residuales (Ward y Savage, 1994; Muhammad et al., 2009; Aslam et al., 2011).

Es importante destacar que en México poco se ha estudiado al respecto y por lo anteriormente mencionado el presente trabajo tuvo como objetivo determinar la calidad nutricional, tipos y concentraciones de metales pesados existentes en la leche de vaca producida en la región irrigada con aguas residuales procedentes del canal de riego de Valsequillo en el estado de Puebla, considerando que esta región ha sido irrigada con estas aguas desde hace más de 40 años. 
MATERIALES Y MÉTODOS

\section{Localización}

La presente investigación se llevó a cabo en la cuenca lechera comprendida por los municipios de Tecamachalco, Tochtepec, San Lorenzo Ometepec, Tlalnepantla y Atoyatempan, ubicados al sureste del estado de Puebla y con clima templado con lluvias en verano a una altitud promedio de $2018 \mathrm{msnm}$ (INEGI, 2015).
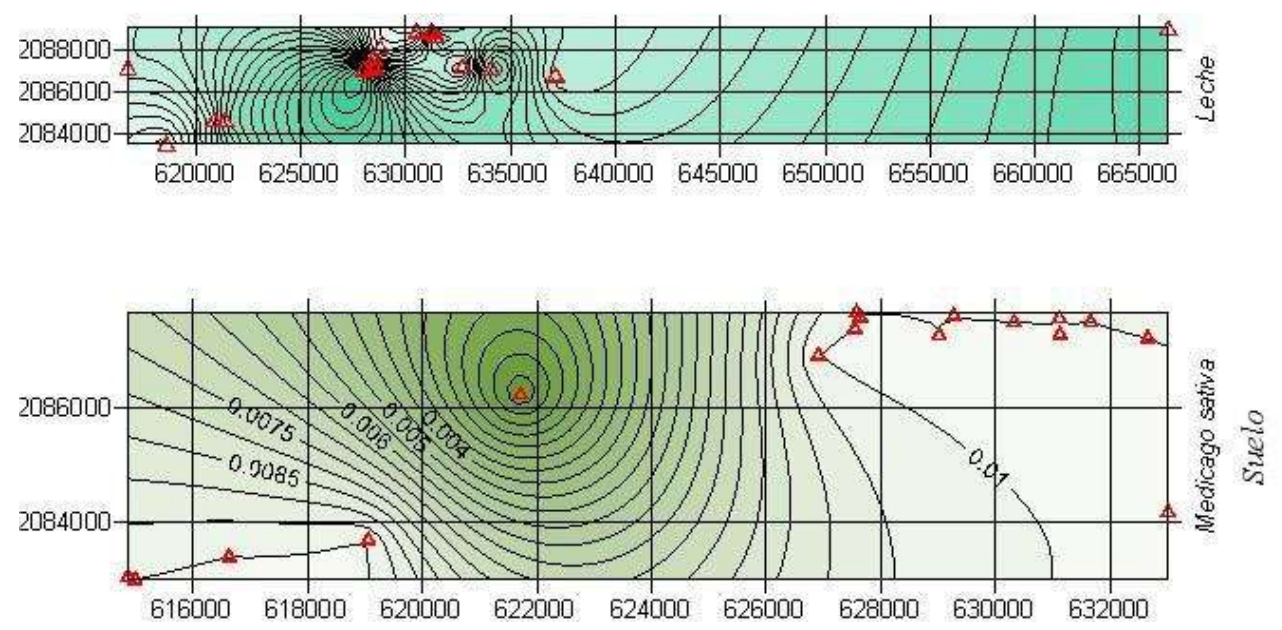

Figura 1.- Coordenadas de los puntos de muestreo de Suelo, Alfalfa (Medicago sativa) y leche.

El trabajo fue realizado en la temporada de lluvias comprendida en los meses de junio y julio de 2014, para lo cual se muestrearon 17 unidades de producción, entre pequeños, medianos y grandes productores, teniendo como característica común la alimentación basada en alfalfa cultivada en suelos irrigados con aguas residuales del canal de riego de Valsequillo.

\section{Suelos agrícolas}

El suelo se colectó en 17 sitios a una profundidad de 0 a $30 \mathrm{~cm}$, tomando $10 \mathrm{sub}$ muestras en cada uno para formar muestras compuestas (Singh et al., 2004). Se colocaron en bolsas de polietileno color negro y se transportaron al laboratorio y fueron secadas al aire libre en un lugar oscuro por 8 días, trituradas, tamizadas a $<2 \mathrm{~mm}$ y guardadas en bolsas hasta su análisis.

\section{Muestreo de alfalfa (Medicago sativa)}

Se colectaron tres plantas de alfalfa por triplicado de los mismos sitios de donde se muestreo el suelo y se trasportaron en bolsas oscuras, fueron lavadas con agua desionizada (Wang et al., 2012) y secadas a $70{ }^{\circ} \mathrm{C}$ por 72 horas (Peralta-Videa et al., 2004), se molieron, tamizaron y almacenaron hasta su digestión y análisis.

\section{Muestreo de leche}

Fueron muestreadas 136 vacas, colectando la leche en dos tubos Falcón de 50 $\mathrm{ml} \mathrm{c} / \mathrm{u}$, previamente lavados con $\mathrm{HNO}_{3}$ al $10 \%$ $v / v$ para determinar metales pesados $\mathrm{y}$ esterilizados para establecer la composición de la leche. Las muestras fueron colectadas al inicio de la ordeña directamente de la ubre posterior al despunte, transportándola en hieleras, realizando el análisis de composición de inmediato en un Milk Analizer Lactoscan 
(Milktronic, Nova Zagora Bulgaria) para evitar cambios en el contenido de la leche y la otra muestra fue congelada a $-65{ }^{\circ} \mathrm{C}$ y liofilizada en un equipo LABCONCO Freezone 4.5 liter (Kansas City, Missouri).

\section{Determinación de metales pesados}

Previo al análisis de metales pesados se realizó la digestión de suelo, leche y alfalfa utilizando para ello un microondas (CEM MarsX, CEM Corporation, Mathews, North Carolina). El suelo se digirió bajo el protocolo EPA 3051 y en el caso de la planta se tomaron $0.3 \mathrm{~g}$ y se agregaron $5 \mathrm{ml}$ de $\mathrm{HNO}_{3}$ ultra puro más $5 \mathrm{ml}$ de Peróxido de hidrógeno al $30 \% \mathrm{w} / \mathrm{v}$. Para la leche se utilizaron $0.5 \mathrm{~g}$ y se agregaron $10 \mathrm{ml}$ de $\mathrm{HNO}_{3}$ ultra puro. Todas las muestras se filtraron utilizando papel Watman 42 y aforaron a $50 \mathrm{ml}$. Para la determinación de metales pesados $\mathrm{Cd}, \mathrm{Pb}, \mathrm{Cr}$ y $\mathrm{Zn}$, se utilizó un espectrofotómetro de absorción atómica $A A S$ Varian 55B. Los productos químicos utilizados eran de grado reactivo analítico. Se prepararon las soluciones en agua desionizada (cero concentración de metal). Los estándares de calibración para cada metal fueron preparados utilizando solución patrón de 1000 ppm de Analitical standar Sigma Aldrich.

\section{Análisis estadístico}

La información obtenida se analizó bajo un diseño completamente al azar mediante un modelo general lineal (GLM) y para la comparación de medias se efectuó la prueba de Tuckey, utilizando el paquete estadístico SAS 9.0 (2002).

\section{RESULTADOS Y DISCUSIÓN}

No existió diferencia $(\mathrm{p} \geq 0.05)$ para la calidad nutricia de la leche por el tamaño de productor. Sin embargo, hay que señalar que la leche tiene baja calidad en cuanto a la cantidad de grasa $(2.02 \%)$, así los sólidos totales presentan un valor bajo (11.06\%). Esto pudo ser determinante en cuanto a la concentración de metales en leche, ya que los metales pesados se adhieren a la grasa debido a un complejo lipofílico formado por la leche, por tanto al ser baja en este componente impide mayores concentraciones de metales pesados (Leeuwen y Pinheiro, 2001; Buechler et al., 2002).

Cuadro 1.- Concentración de metales pesados en suelo irrigado con aguas del canal de Valsequillo Puebla, México 2014.

\begin{tabular}{ccc}
\hline Metales pesados & Suelo $\left(\mathrm{mg} \mathrm{kg}^{-1}\right)$ & NMX-001-ECOL-1996 \\
\hline $\mathrm{Cd}$ & 2 & 0.05 \\
$\mathrm{~Pb}$ & 38 & 5 \\
$\mathrm{Cr}$ & 31.38 & 0.5 \\
$\mathrm{Zn}$ & 22.78 & 10 \\
\hline
\end{tabular}


Cuadro 2.- Concentración de metales pesados $\left(\mathrm{mg} \mathrm{kg}^{-1}\right)$ en alfalfa irrigada con agua del canal de Valsequillo, Puebla, México 2014.

\begin{tabular}{ccc}
\hline & $\begin{array}{c}\text { Alfalfa (Medicago sativa) } \\
\text { Metales pesados }\end{array}$ & ( $\left.\mathrm{mg} \mathrm{kg}^{-1}\right)$ \\
\hline $\mathrm{Cd}$ & 0.8 & 0.5 \\
$\mathrm{~Pb}$ & 13.39 & 10 \\
$\mathrm{Cr}$ & 2.15 & - \\
$\mathrm{Zn}$ & 8.65 & 50 \\
\hline
\end{tabular}

*LPM (límite máximo permisible)

Las concentraciones de metales pesados en el suelo, están por arriba de los límites permisibles de la NMX-001-ECOL-1996 (cuadro 1), esto es debido a que la región es irrigada dos veces por año desde hace aproximadamente 40 años y se sabe que debido al tiempo de uso del agua residual se aumenta el grado de contaminación de los suelos y los cultivos, lo que amplía el grado de toxicidad (Chary et al., 2008; Singth et al., 2010).

Así mismo la planta mostró concentraciones elevadas de metales pesados, sin embargo no existen datos en la norma mexicana para hacer comparación, por ello se utilizó la norma de la (WHO 1995) (cuadro 2) con lo cual se pudo determinar que existen niveles por arriba de dicha norma en cuanto a $\mathrm{Cd} \mathrm{y} \mathrm{Pb}$, pudiéndose comprobar que la concentración de metales en suelos incide directamente en la cadena alimenticia, siendo la alfalfa el alimento base de los bovinos productores de leche en la región de estudio. La absorción y bioacumulación que realiza la alfalfa es el primer paso para el ingreso de los metales pesados a la cadena alimenticia y algunos metales son más fitodisponibles que otros (Kabata y Pendias, 2000) lo que genera riesgo de salud y de seguridad alimentaria.

En el caso de la leche la concentración de $\mathrm{Pb}$ (cuadro 3) es más alta que el límite permisible por la WHO/FAO y el código de la Unión Europea, y con respecto al Cd, Cr, y Zn no están considerados dentro de la norma mexicana y la europea, sin embargo de acuerdo con la norma brasileña el nivel de $\mathrm{Cd}$ en este trabajo está por debajo de dicha norma y el $\mathrm{Cr}$ por el contrario se encontró en un nivel más alto.

Cuadro 3.- Concentración de metales pesados en leche de vacas alimentadas con alfalfa irrigada con aguas del canal de Valsequillo, Puebla, México 2014.

Norma

NOM-243- Unión oficial

\begin{tabular}{cccccc} 
Metales pesados & Leche $\left(\mathrm{mg} \mathrm{kg}^{-1}\right)$ & SSA1-2010 & Europea & WHO/FAO & Brasil \\
\hline $\mathrm{Cd}$ & 0.01 & -- & -- & -- & 1 \\
$\mathrm{~Pb}$ & 0.13 & 0.1 & 0.02 & 0.02 &
\end{tabular}

Revista Científica Biológico Agropecuaria Tuxpan 4 (1) 

$\mathrm{Cr}$
0.4
$\mathrm{Zn}$
0.18

Con relación a la norma mexicana NOM-243-SSA1-2010, la concentración de Pb encontrada en este trabajo está ligeramente por encima del límite permisible (cuadro 3), sin embargo, hay que hacer mención de que en esta norma se considera únicamente leche pasteurizada y no la leche cruda. El nivel de $\mathrm{Pb}$ y $\mathrm{Cr}$ encontrado en este trabajo es inferior al reportado en Pakistán por Aslam et al. (2011) donde el nivel de $\mathrm{Pb}$ fue 23.24 y el de $\mathrm{Cr} 1.3$ $\mathrm{mg} \mathrm{kg}^{-1}$. En cuanto al $\mathrm{Zn}$ se encontró un nivel por debajo de lo obtenido por Moreno-Rojas et al. (1994) quienes determinaron $3.5 \mathrm{mg} \mathrm{kg}^{-1}$ en España. Con respecto al $\mathrm{Cd}$, el valor conseguido en este trabajo es inferior del obtenido por Kazi et al. (2009) y Singh et al. (2010) quienes reportan 44.2 y $0.044 \mathrm{mg} \mathrm{kg}^{-1}$ en Pakistán y la India respectivamente.

Al hacer la comparación entre concentraciones por matriz se observó que existe $\mathrm{p} \leq 0.0001$ entre suelo, planta (Medicago sativa) y leche, siendo esta última la de menor concentración. Sin embargo, esto demuestra que los metales pesados tienen movilidad en la cadena alimenticia, lo que convierte a los suelos en un medio importante de contaminación de los cultivos y estos últimos a la leche (Gulson et al., 1996).

\section{CONCLUSIONES}

De acuerdo a los resultados obtenidos se concluye que la calidad en cuanto a la composición de la leche en la región estudiada es baja y esto puede deberse a las malas prácticas de manejo nutricional aplicadas al ganado. Por otra parte la concentración de metales pesados encontrados es alto, principalmente en lo concerniente al $\mathrm{Pb}$ y $\mathrm{Cr}$ lo que implica un grave riesgo de salud, principalmente para los niños y por ello se debe realizar un monitoreo constante y tomar medidas para el control de la contaminación por medio de programas de biorremediación de los suelos así como realizar análisis de riesgo a la salud pública.

\section{LITERATURA CITADA}

Al-Othman Z.A., Naushad, M, Khan M.R., Wabaidur S.M. 2012. A comparative study on characterization of aluminium tungstate and surfactant based aluminum tungstate cation exchangers: analytical applications for the separation of toxic metal ions. J. Inorg. Organomet. Polym. 22, 352-359. https://doi.org/10.1007/s10904-011-9594 Álvarez-Fuentes G., Herrera-Haro J. G, AlonsoBastida G., Barreras-Serrano A. 2012. Calidad de leche cruda en unidades de producción al sur de la Ciudad de México. Arch. Med. Vet. 44:237-242. https://doi.org/10.4067/S030

Aslam B., Ijaz Javed, Faqir Hussain Khan, and Zia Ur Rahman. 2011. "Uptake of Heavy Metal Residues from Sewerage Sludge in the Milk of Goat and Cattle during Summer Season." Pakistan Veterinary Journal 31: 75-77.

ATSDR. Toxicological profile for lead. US Department of Health and Human Services, Public Health Service, Agency for Toxic Substances and Disease Registry, Atlanta, GA. 2007 [citado 01 de abril 2013]. Disponible en: URL:http://www.atsdr.cdc.gov/substances/ toxsubstance. asp?toxid=15

Bobić T., Mijić, P., Gregić, M., Ivkić, Z., Baban, M. 2013. The influence of ordinal number and stage of lactation on milkability traits in Holstein cows, Mljek-arstvo 63 (3), 172-179.

Buechler S.J., Devi, G., Raschid-Sally, L., 2002. Livelihoods and wastewater 
irrigated agriculture along the Musi River in Hyderabad City, Andhra Pradesh, India. U. A. Magazine 8, 1417.

Chary N S., C T Kamala, and D Samuel Suman. 2008. "Assessing Risk of Heavy Metals from Consuming Food Grown on Sewage Irrigated Soils and Food

Chain Transfer" 69: 513-24. https://doi.org/10.4067/S0301-732X201

Diario oficial de la Unión Europea disponible en; http://eur-lex.europa.eu/legalcontent/ES/TXT/PDF/?uri=OJ:L:2014:1 04:FULL\&from=ES, 07 de junio de 2015.

Ducháček J., Stádník, L., Ptáček, M., Beran, J., Okrouhlá, M., Č́tek, J., Stupka, R. 2014. Effect of cow energy status on hypercholesterolemic fatty acids proportion in raw milk, Czech Journal of Food Science 32 (3), 273-279.

https://doi.org/10.17221/360/2013-CJFS

Doreaa J. G. y Donangelo C. M. 2006. Early (in uterus and infant) exposure to mercury and lead. Clinical Nutrition 25,369376.

https://doi.org/10.1016/j.clnu.2005.10.00

FAO. 2014. CODEX STAND 193-195. Disponible en: http://www.fao.org/fileadmin/user_uplo ad/livestockgov/documents/CXS_193s.p df

Gulson B.L., K.J. Mizon., M.J. Korsch. \& D. Howarth. 1996. Non-orebody sources are significant contributors to blood lead of some children with low to moderate lead exposure in a mayor mining community. The science of the total environment. 181: 223-230.

https://doi.org/10.1016/0048-9697(95)05

INEGI, Instituto Nacional de Estadística Geografía e Informática. 2015. Disponible en: http://www.inegi.org.mx/

INEGI/COLPOS. 1999. La ganadería familiar en México. Instituto Nacional de Estadística Geografía e Informática, Colegio de Postgraduados. México.
Kabata-Pendias A. 2000. Trace elements in soils and plants. Third Edition. CRC Press, Inc. Boca Raton. USA. pp. 365, 413. https://doi.org/10.1201/9781420039900

Kazi T. G., Jalbani N, Baig, J. A, Kandhro G A, Afridi H. I, Arain M. B, Jamali M. K, and Shah A. Q. 2009. "Assessment of Toxic Metals in Raw and Processed Milk Samples Using Electrothermal Atomic Absorption Spectrophotometer." Food and Chemical Toxicology 47 (9). Elsevier Ltd: 2163-69. https://doi.org/10.1016/j.fct.2009.05.035 Kadlecová V., Němečková, D, Ječmínková, K, Stádník, L. 2014. The effects of polymorphism in the DGAT1 gene on energy balance and milk production traits in primiparous Holstein cows during the first six months of lactation, Bulgarian Journal of Agricultural Science 20 (1), 203-209.

Leeuwen H.P.V., Pinheiro, J.P. 2001. Speciation dynamics and bioavailability of metals. Exploration of the case of two uptake routes. Pure Appl. Chem. 73 (1), 39-44.

https://doi.org/10.1351/pac20017301003

Moreno-Rojas R., Amaro-Lopez, M. a., ZureraCosano, G., 1994. Copper, iron and zinc variations in Manchego-type cheese during the traditional cheese-making process. Food Chem. 49, 67-72. https://doi.org/10.1016/0308-8146(94)90

Muhammad, F, M Akhtar, I Javed, Z U

Rahman, I Jan, M I Anwar, and S Hayat. 2009. Quantitative Structure Activity Relationship and Risk Analysis of Some Heavy Metal Residues in the Milk of Cattle and Goat. Toxicology and Industrial Health 25 (3): 177-81.

https://doi.org/10.1177/074823370910559

NOM-001-ECOL-1996. Establece los límites máximos permisibles de contaminantes en las descargas de agua residuales en aguas y Bienes Nacionales. Diario Oficial de la Federación (DOF) 1997. 
NOM-243-SSA1-2010. Productos y servicios. Leche, fórmula láctea, producto lácteo combinado y derivados lácteos.

Disposiciones y especificaciones sanitarias. Métodos de prueba. Disponible en: http://dof.gob.mx/nota_detalle.php?codi go=5160755\&fecha $=27 / 09 / 2010$

Oliver M A, 1997. Soil and human health: a review. European Journal of Soil Science, $\quad 48(4)$ : 573-592. https://doi.org/10.1046/j.1365-2389.1997

Peralta-Videa J. R.., G. de la Rosa, J. H. Gonzalez, and J. L. Gardea-Torresdey. 2004. "Effects of the Growth Stage on the Heavy Metal Tolerance of Alfalfa Plants." Advances in Environmental https://doi.org/10.1016/S1093-0191(03)0

Rego O.A., Alves, S.P., Antunes, L.M., Rosa, H.J., Al-faia, C.F., Prates, J.A., Carita, A.R., Fonseca, A.J., Bessa, R.J. 2009. Rumen biohydrogenation-derived fatty acids in milk fat from grazing dairy cows supplemented with rapeseed, sunflower, or linseed oils, Journal of Dairy Science 92 (9), 4530-4540. https://doi.org/10.3168/jds.2009-2060

SAS, Institute. 2002. SAS User's Guide: Statistics Version 9.2. Statistical Analysis System Institute Cary, North Carolina, USA.

Singh A., Rajesh KS, Agrawal M,. Marshall F. M. 2010. Health risk assessment of heavy metals via dietary intake of foodstuffs from the wastewater irrigated site of a dry tropical area of India. Food and Chemical Toxicology 48 (2010) 611-619.

https://doi.org/10.1016/j.fct.2009.11.041
Singh K.P., Mohan, D., Sinha, S., Dalwani, R. 2004. Impact assessment of treated/untreated wastewater toxicants discharged by sewage treatment plants on health, agricultural, and environmental quality in the wastewater disposal area. Chemosphere 55, $227 \mathrm{e} 255$. https://doi.org/10.1016/j.chemo

Soares V., Mahyara M M Kus, André Luis C Peixoto, Juliana S. Carrocci, Rodrigo F S Salazar, and Hélcio J. Izário Filho. 2010. "Determination of Nutritional and Toxic Elements in Pasteurized Bovine Milk from Vale Do Paraiba Region (Brazil)." Food Control 21 (1). Elsevier Ltd: 45-49. https://doi.org/10.1016/j.foodcont.2009. Sundberg J., Jo“nsson S., Karlsson M. O., and A. Oskarsson. 1999. Lactational Exposure and Neonatal Kinetics of Methylmercury and Inorganic Mercury in Mice. Toxicology and Applied Pharmacology 154, 160-169. https://doi.org/10.1006/taap.1998.8566

Ward NI and JM Savage. 1994. Metal dispersion and transportational activities using food crops as biomonitors. Sci Total Environ, 146: 309-319. https://doi.org/10.1016/0048-9697(94)90

Wang Y., Qiao M., Liu Y., Zhu Y. 2012. Health risk assessment of heavy metals in soils and vegetables from wastewater irrigated area, Beijing-Tianjin city cluster, ChinaJournal of Environmental Sciences, 24(4) 690-698. https://doi.org/10.1016/S1001-0742(11)6 0833-4 


\section{Copyright (c) 2016 Numa Pom pilio Castro G onzález, Rafael Moreno Rojas, Francisco Calderón Sánchez, \\ Ramiro Escobar Hernández y Benjamin Barrios Diaz}

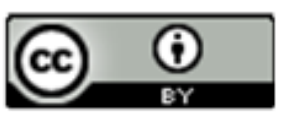

Este tex to está protegido por una licencia licencia CreativeCommons 4.0.

Usted es libre para Compartir —copiar y redistribuir el material en cualquier medio o form ato-y Adaptar el documento —remezclar, transformar y crear a partir del material- para cualquier propósito,, incluso para fines comerciales, siempre que cumpla la condición de:

Atribución: Usted debe dar crédito a la obra original de manera adecuada, proporcionar un enlace a la licencia, e in dicar si se han realizado cam bios. Puede hacerlo en cualquier form a razonable, pero no de form a tal que sugiera que tiene el apoyo del licenciante o 10 recibe por el uso que hace de la obra.

Resumendelicencia - Textocompletodelalicencia 